\title{
Linfangiomatosis mesentérica como causa de abdomen agudo en la tercera edad*
}

\author{
Drs. FELIPE LEÓN F. ${ }^{1}$, GUSTAVO PÉREZ B. ${ }^{1,}$ GONZALO MÉNDEZ O. ${ }^{2}$, CLAUDIA BOLADOS A. ${ }^{2}$ \\ 1 Departamento de Cirugía Digestiva. \\ 2 Departamento de Anatomía Patológica. \\ Pontificia Universidad Católica de Chile. Santiago, Chile.
}

\begin{abstract}
Mesenteric lymphangiomathosis presenting as an acute abdomen in an older subject

We report a 71 years old male who consulted in the emergency room for abdominal pain lasting 12 hours. On physical examination there was pain on abdominal palpation and signs of peritoneal irritation. An abdominal CT scan showed a thickening of the medial and distal ileum, multiple adjacent collections and signs of peritonitis. The patient was operated, observing multiple white tumors in the mesentery and serosa of small bowel, one of these lesions, adhered to the bowel wall, had a hemorrhagic infarct. One of the lesions was punctured, obtaining a milky fluid. Biopsies were obtained and the infarcted lesion was excised. The pathological study reported a Mesenteric Multilocular Lymphangioma. The patient had an uneventful postoperative evolution.
\end{abstract}

Key words: Lymphangioma, mesenteric, acute abdomen.

\section{Resumen}

Presentamos el caso clínico de un hombre de 71 años, sin antecedentes mórbidos, salvo apendicectomía y colecistectomía hace más de 20 años. Consulta en servicio de urgencias por dolor abdominal de 12 horas de evolución, sin otros síntomas asociados. Al examen físico destacaba dolor abdominal e irritación peritoneal a la palpación de hipocondrio izquierdo. Exámenes destacan aumento de parámetros inflamatorios, la tomografía computada de abdomen y pelvis revela engrosamiento de íleon medio-distal, con múltiples colecciones adyacentes y signos de peritonitis. Se realiza laparotomía exploradora, evidenciándose múltiples tumoraciones blanquecinas en mesenterio y serosa de todo el intestino delgado, una de las cuales se aprecia con infarto hemorrágico adherida a la pared abdominal. Punción de lesiones da salida a líquido lechoso. Se toman biopsias y se reseca lesión infartada. Paciente evoluciona favorablemente, dado de alta al tercer día. La histología reveló un Linfangioma Quístico Multilocular Mesentérico. Pese a ser una patología infrecuente, debe ser considerada dentro de los diagnósticos diferenciales de abdomen agudo, siendo extremadamente rara su presentación en la tercera edad.

Palabras clave: Linfangioma, mesenterio, abdomen agudo.

*Recibido el 7 de diciembre de 2011 y aceptado para publicación el 29 de febrero de 2012.

Los autores no declaran conflictos de interés.

Correspondencia: Dr. Felipe León F.

Marcoleta 352, Santiago. Chile. Fax: 56-2-3543462

felipeleon81@gmail.com 


\title{
Introducción
}

Los linfangiomas son lesiones de origen linfático, benignos e infrecuentes, que se suelen presentar en la infancia o adolescencia, habitualmente en menores de 20 años. Su presentación en la tercera edad es extremadamente rara. Se localizan habitualmente en la piel de cabeza, cuello o axila, siendo la localización intrabdominal menor a un $5 \%$ de los casos. Presentan una relación hombre:mujer de 3:1 $1^{1}$.

La presentación clínica es variable, siendo en la mayoría de los casos asintomáticos o manifestándose por dolor abdominal vago. Existen algunos reportes en la literatura de su debut como abdomen agudo o hallazgos en laparotomía por otras causas ${ }^{2}$. El término linfangiomatosis se utiliza para definir los linfangiomas con afectación multifocal o difusa de órganos o partes blandas ${ }^{3}$. El pronóstico de esta enfermedad viene determinado por la extensión de las lesiones, dependiendo de esto último la posibilidad de exéresis quirúrgica completa ${ }^{4}$.

Nuestro objetivo es presentar el caso de un paciente adulto mayor que consulta en nuestro servicio de urgencia con un cuadro clínico de abdomen agudo, cuya causa fue una Linfangiomatosis Mesentérica complicada. Además se realiza una revisión de la literatura al respecto.

\section{Caso clínico}

Consulta en Servicio de Urgencia un paciente de sexo masculino de 71 años de edad, con antecedentes de colecistectomía hace 30 años y apendicectomía hace 20 años en otro hospital. No presenta otros antecedentes mórbidos. Refiere cuadro de dolor abdominal de 12 horas de evolución en flanco e
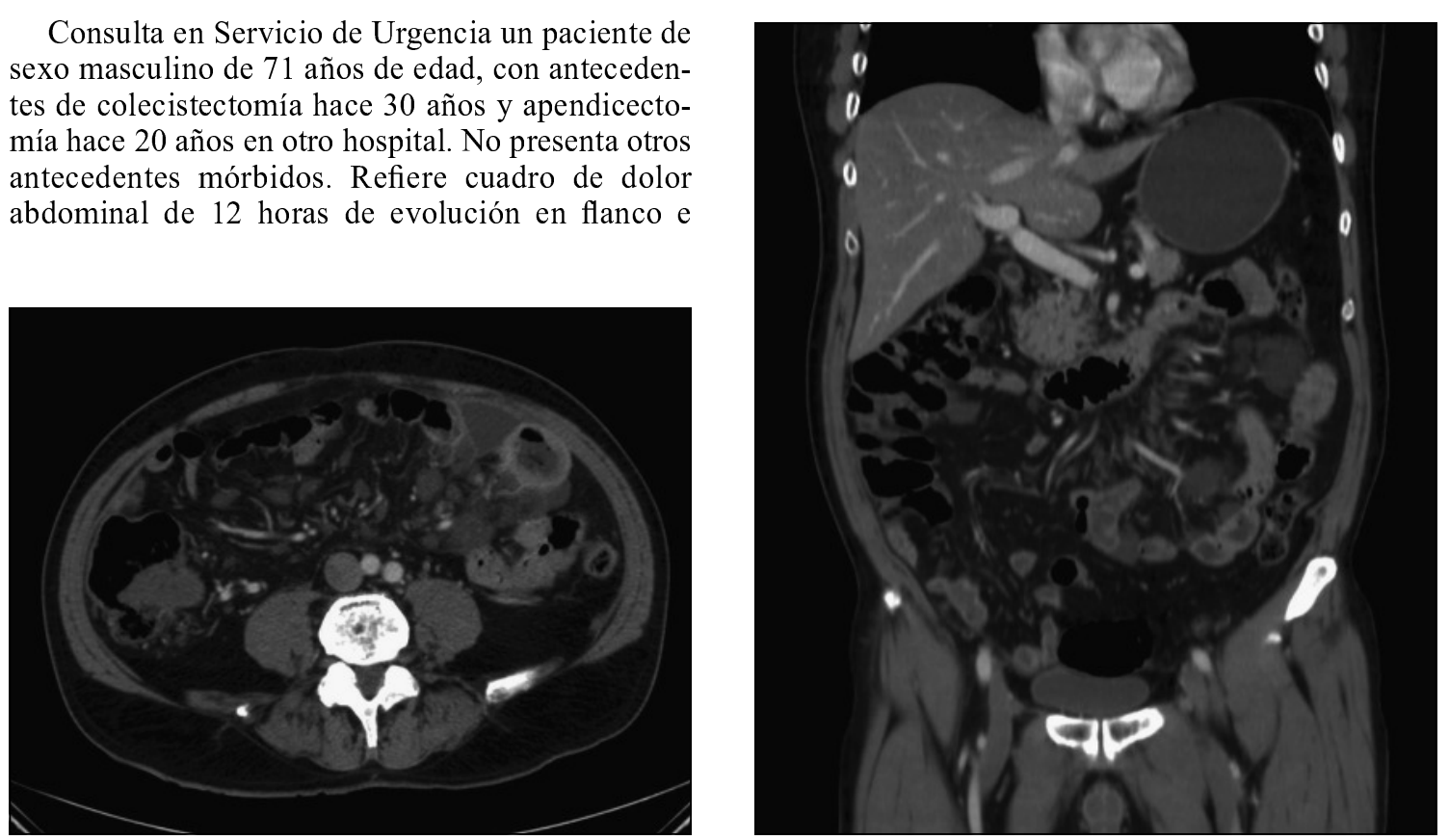

Figuras 1 y 2. Engrosamiento aislado de asas de íleon medio-distal, con múltiples colecciones adyacentes y en hojas del mesenterio, junto con signos de peritonitis.

hipocondrio izquierdo, de inicio súbito e intensidad severa, sin otros síntomas asociados. Al examen físico destacaba afebril, normotenso, sin taquicardia. $\mathrm{Al}$ examen abdominal presentaba dolor exquisito a la palpación de hipocondrio izquierdo, con signos de irritación peritoneal a este nivel. No se palpan masas ni otros hallazgos. Se solicitan exámenes: Hematocrito: 41\%, Leucocitos: 7.400, Proteína C Reactiva: $122 \mathrm{mg} / \mathrm{dl}$ (límite normal de 10), Creatinina: $1,01 \mathrm{mg} / \mathrm{dl}$. Amilasa: normal. Se decide realizar Tomografia Axial Computada de abdomen y pelvis (Figuras 1 y 2) sugerentes de múltiples colecciones intraabdominales y engrosamiento de algunas asas de intestino delgado, junto con signos de peritonitis secundaria.

Se decide realizar laparotomía exploradora (Figuras 3 y 4), en la cual se evidencian múltiples tumoraciones blanquecinas en mesenterio y serosa de todo el intestino delgado, una de las cuales se aprecia con infarto hemorrágico y adherida a la pared abdominal de hipocondrio izquierdo. Punción de las lesiones da salida a líquido lechoso. Se realiza biopsia de algunas lesiones y se reseca lesión infartada, junto con aseo de la cavidad abdominal. No se evidencia perforación de víscera hueca ni otros hallazgos.

El paciente evoluciona favorablemente con tratamiento antibiótico biasociado (CeftriaxonaMetronidazol), siendo realimentado en forma progresiva y dado de alta al tercer día postoperatorio en

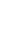




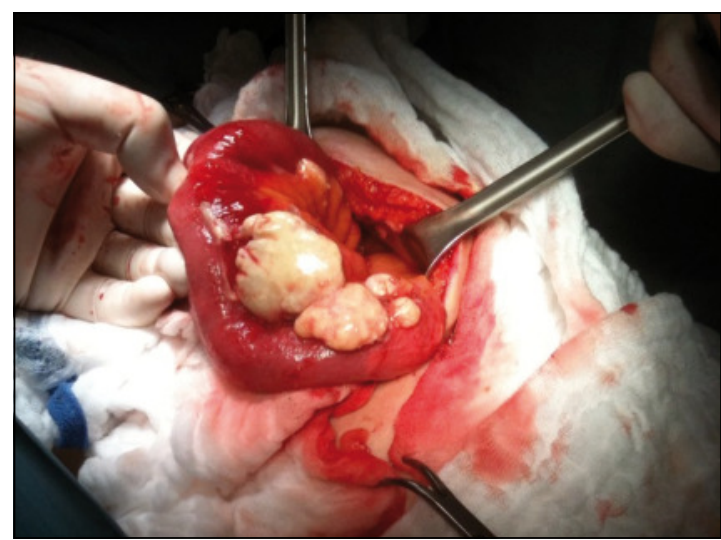

Figura 3. Múltiples tumoraciones blanquecinas en mesenterio y serosa de todo el intestino delgado. Punción de lesiones da salida a líquido lechoso.

buenas condiciones, con escaso dolor abdominal. Se deja tratamiento ambulatorio con antibióticos orales y analgésicos. Al interrogar al paciente de manera dirigida, refiere que su cirujano tratante observó los mismos hallazgos durante ambas laparotomías previas hace más de 20 años, sin realizarse más estudio posterior debido a la nula sintomatología abdominal.

El informe anatomopatológico reveló un Linfangioma Quístico Multilocular Mesentérico, con hiperplasia mesotelial de tipo reactivo perifocal (Figura 5).

Debido a la gran extensión de la enfermedad, se decide en conjunto con el paciente realizar controles periódicamente y consultar en caso de síntomas. A un año de la cirugía, el paciente se mantiene en buenas condiciones, asintomático y asistiendo a controles en forma seriada.

\section{Discusión}

La etiología de los linfangiomas aún es motivo de discusión. Son considerados una displasia congénita del tejido linfático, cuyo origen sería un anormal desarrollo de los vasos linfáticos durante la vida fetal, produciendo un secuestro localizado de tejido linfático a este nivel ${ }^{5,6}$. Sin embargo, existen algunas teorías que involucran a factores desencadenantes del anormal desarrollo linfático, tales como trauma, obstrucción linfática, procesos inflamatorios, cirugía y radioterapia. Estos factores serían potenciales desencadenantes de esta patología, explicando su presentación tardía en algunos adultos que incluso poseían exámenes radiológicos normales previos ${ }^{1}$.

Los linfangiomas mesentéricos son extremadamente raros, siendo el motivo de hospitalización en 1:20.000 a 1:250.000 de las admisiones hospi-

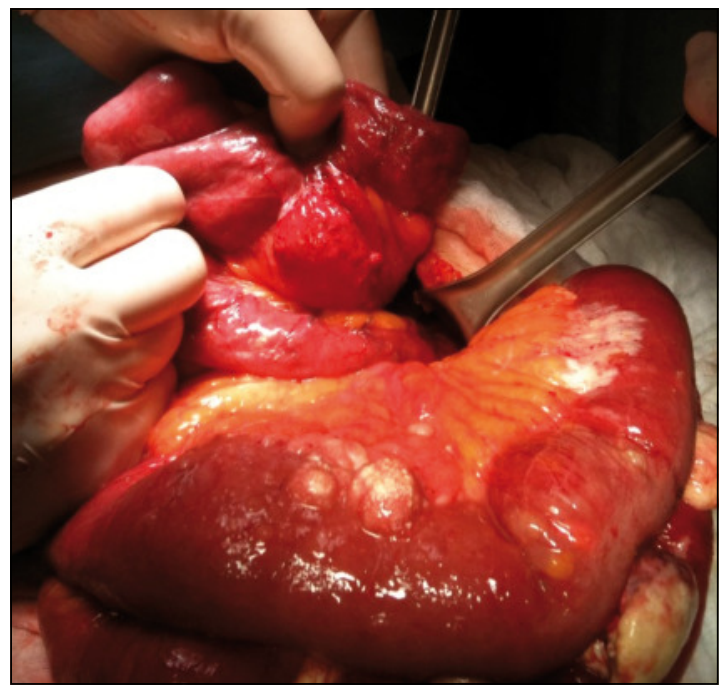

Figura 4. Infarto hemorrágico de linfangioma adherido a pared abdominal de hipocondrio izquierdo.

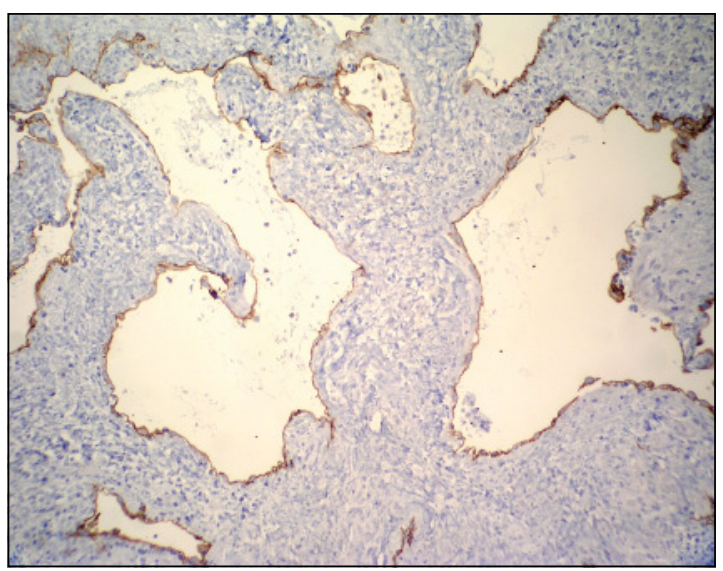

Figura 5. Corte histológico de zona quística multilocular, formada por vasos linfáticos de tamaño variable e hiperplasia mesotelial de tipo reactivo perifocal adyacente (Estudio inmunohistoquímico D2-40; 100X).

talarias 7 . Del total de linfangiomas, sólo un 5\% se presentan bajo el diafragma, en un $63 \%$ de los casos involucrando el lado izquierdo del cuerpo ${ }^{2}$. En el abdomen involucran principalmente el retroperitoneo, mesenterio y mesocolon, junto con algunos órganos sólidos (bazo e hígado). Dentro de su localización intraperitoneal, afectan mayormente al mesenterio del intestino delgado en un $70 \%$ de los casos, siendo la ubicación ileal la más frecuente con un 50 a $60 \%$ de los casos. La afectación del mesenterio puede ser focal o difusa ${ }^{8}$.

Estas lesiones son clasificadas en tres categorías desde el punto de vista histológico: simples, caver- 
nosos y císticos, pudiendo encontrarse más de un tipo histológico en el mismo tumor 9 . Macroscópicamente son lesiones quísticas de paredes delgadas, de color amarillento, con múltiples tabiques en su interior, los cuales los dividen en cavidades irregulares de variados tamaños comunicadas entre sí. Usualmente estas cavidades se encuentran llenas de líquido con alto contenido proteico y eosinófilos, de aspecto lechoso ${ }^{10}$. Dentro del diagnóstico diferencial histológico están los mesoteliomas, algunos tumores pancreáticos y los hemangiomas cavernosos cuando las lesiones han presentado hemorragia ${ }^{1}$. En nuestro caso clínico el principal diagnóstico diferencial lo constituyó el mesotelioma quístico, siendo realizado el diagnóstico definitivo con los hallazgos histopatológicos y estudio con inmunohistoquímica D2-40.

Los linfangiomas de localización intraabdominal habitualmente son asintomáticos. La mayoría de las veces se manifiestan como masa abdominal indolora (linfangiomas quísticos) o dolor abdominal vago, pero hasta en el $25 \%$ de los casos pueden debutar como abdomen agudo ${ }^{11}$. Dependiendo de su crecimiento y localización, pueden afectar diversas estructuras intraabdominales, provocando obstrucción mecánica de asas intestinales, uréteres o vasculares. La infiltración de órganos y estructuras vecinas puede provocar hemorragia intraabdominal, perforación de víscera hueca, infarto, torsión de vísceras o vólvulos intestinales ${ }^{11,12}$. Además existe la posibilidad de sobreinfectarse de forma secundaria ${ }^{12,13}$. Debido a lo anterior, pese a ser lesiones benignas pueden presentar múltiples complicaciones y morbi-mortalidad importante $^{14,15}$.

El estudio por imágenes revela en la ultrasonografía lesiones quísticas bien definidas con múltiples tabiques en su interior o lesiones hipoecogénicas con refuerzo acústico posterior ${ }^{16}$. Los hallazgos a la tomografia axial computada muestran característicamente masas uni o multiloculares que contienen septos de grosor variable; con el contraste se observa refuerzo en la pared y permite precisar la anatomía del linfangioma, localización, extensión y relación con los órganos vecinos. Muchas veces estas lesiones se pueden confundir con colecciones intraabdominales, tal como sucedió en nuestro caso, haciendo difícil el diagnóstico preciso preoperatorio. La resonancia nuclear magnética es el instrumento más adecuado para el diagnóstico y caracterización preoperatoria, permitiendo la planificación quirúrgica de manera más adecuada. Permite además diferenciar los quistes mesentéricos de los linfangiomas, al definir adecuadamente la presencia de estructuras quísticas y tabiques en su interior ${ }^{17}$.

El tratamiento de elección de esta patología es la remoción quirúrgica completa de las lesiones, incluso en pacientes asintomáticos ${ }^{18,19}$. Cualquier resección incompleta se asocia a un índice de recidivas del 10 a $15 \%$ de los casos, al contrario de la casi nula recidiva cuando la remoción es completa ${ }^{20}$. En caso de no ser posible la resección completa por la extensión o localización de la enfermedad, se han descrito numerosos tratamientos tales como la escleroterapia con doxiciclina o alcohol ${ }^{21}$ y la radioterapia $^{22}$. Actualmente, se recomiendan además la bleomicina y el OK-432 como soluciones esclerosantes en caso de lesiones mesentéricas técnicamente irresecables ${ }^{23}$.

En conclusión los linfangiomas intraabdominales en adultos son lesiones poco frecuentes, ubicados habitualmente en el mesenterio del intestino delgado. La mayoría de las veces son asintomáticos, pero pueden debutar como abdomen agudo quirúrgico, siendo a veces un hallazgo intraoperatorio. El diagnóstico es difícil, sobretodo en pacientes mayores, debiendo ser considerado dentro del diagnóstico diferencial de abdomen agudo. Debe ser confirmado siempre con la histopatología. El tratamiento de elección es la remoción completa de las lesiones, existiendo en la actualidad otras alternativas no quirúrgicas en caso de no ser técnicamente posible la extirpación en su totalidad.

\section{Referencias}

1. Rieker R, Quentmeier A, Weiss C, Kretzschmar U, Amann K, Mechtersheimer G, et al. Cystic Lymphangioma of the Small Bowel Mesentery: Case Report and a Review of the Literature. Pathol Oncol Res. 2000;6:146-8.

2. Wani I. Mesenteric Lymphangioma in Adult: A Case Series with a Review of the Literature. Dig Dis Sci. 2009;54:2758-62.

3. Kably A, Moumen M, Raissouni N, Benjelloun N, Mehhane M, Abi F, et al. Le lymphangiome kystique du mésentère et de l'épiploon. À propos de deux cas. Gynecol Obstet Fertil. 2003;31:136-8.

4. Lara C, Borrero J, Porras V, de la Rosa J.A, Expósito F. Linfangiomatosis Mesentérica. Presentación de un caso. Arch Esp Urol. 2006;59:542-4.

5. Hwang SS, Choi HJ, Park SY. Cavernous mesenteric lymphangiomatosis mimicking metastasis in a patient with rectal cancer: A case report. World J Gastroenterol. 2009;15,31:3947-9.

6. Abe H, Kubota K, Noie T, Bandai Y, Makuuchi M. Cystic lymphangioma of the pancreas: a case report with special reference to embryological development. Am J Gastroenterol. 1997; 92:1566-7.

7. Kurtz MD, Heimann TM, Beck AR, Holt J. Mesenteric and retroperitoneal cysts. Ann Surg. 1986;203:109-12.

8. Chin S, Kikuyama S, Hashimoto T, Tomita T, Hasegawa 
F. LEÓN F. y cols.

T, Ohno Y. Lymphangioma of the jejunal mesentery in an adult: a case report and a review of the Japanese literature. Keio J Med. 1993;42:41-3.

9. Losanoff J, Richman BW, El-Sherif A, Rider KD, Jones J. Mesenteric cystic lymphangioma. J Am Coll Surg. 2003;196:598-603.

10. Hornick JL, Fletcher CD. Intraabdominal cystic lymphangiomas obscured by marked superimposed reactive changes: clinicopathological analysis of a series. Hum Pathol. 2005;36:426-32.

11. Losanoff JE, Kjossev KT. Mesenteric cystic lymphangioma: unusual cause of intra-abdominal catastrophe in an adult. Int J Clin Pract. 2005;59:986-7.

12. Porras-Ramírez G, Hernández-Herrera MH. Hemorrhage into mesenteric cyst following trauma as a cause of acute abdomen. J Pediatr Surg. 1991;26:847-8.

13. Ricca RJ. Infected mesenteric lymphangioma. N Y State J Med. 1991;91:359-61.

14. Griffa B, Basilico V, Feltri M, Griffa A. Submucosal jejunal lymphangioma: an unusual case with obscure gastrointestinal bleeding in an adult, detected by videocapsule endoscopy and treated by laparoscopy. Minerva Chir. 2006;61:529-32.

15. de Vries JJ, Vogten JM, de Bruin PC, Boerma D, van de Pavoordt HD, Hagendoorn J. Mesenterical lymphangiomatosis causing volvulus and intestinal obstruction.
Lymphat Res Biol. 2007;5:269-73.

16. Mostofian E, Ornvold K, Latchaw L, Harris RD. Prenatal sonographic diagnosis of abdominal mesenteric lymphangioma. Ultrasound Med. 2004;23:129-32.

17. Woo K, Jeong YK, Song Y, Heo JN, Park CK. Cavernous mesenteric lymphangioma. Eur Radiol. 2006;16:1625-8.

18. Poon MC-M, Lee DWH, Wong PK, Chan ACW. Mesenteric cystic lymphangioma presented with small bowel volvulus. Ann Coll Surg Hong Kong 2001;5:127-8.

19. Su CM, Yu MC, Chen HY, Tseng JH, Jan YY, Chen MF. Single centre results of treatment of retroperitoneal and mesenteric cystic lymphangioma. Dig Surg. 2007;24:181-5.

20. Pang LC. Acute abdominal conditions in mesenteric lymphangioma. South Med J. 1990;83:467-70.

21. Stein M, Hsu R, Schneider P, Ruebner B, Mina Y. Alcohol ablation of a mesenteric lymphangioma. J Vasc Radiol. 2000;11:247-50.

22. Tai PTH, Jewell LD. Mesenteric mixed haemangioma and lymphangioma; report of a case with 10 year follow-up after radiation treatment. Br J Radiol. 1995;68:657-61.

23. Acevedo JL, Shah RK, Brietzke SE. Nonsurgical therapies for lymphangiomas: a systematic review. Otolaryngol Head Neck Surg. 2008;138:418-24. 